\title{
Robust Target Localization in the Absence of Signal Propagation Models *
}

\author{
Er-wei Bai ${ }^{a}$ and Li Ding ${ }^{b}$

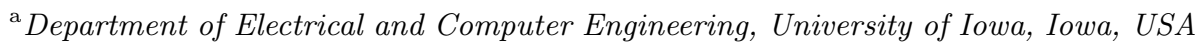 \\ ${ }^{\mathrm{b}}$ Department of Automation, School of Power and Mechanical Engineering, Wuhan University, Wuhan, China
}

\begin{abstract}
This paper considers a problem of target or source localization based on the measurements from a mobile sensor or a network of sensors. The assumption is that the received signal strength is strictly monotonic with the distance between the target and the sensor. No explicit signal propagation model, neither the structure nor the mathematical description, is assumed or used in localization. The contribution of the paper is to show that this mere knowledge of monotonicity suffices in locating the unknown target in the absence of noise. Further in the presence noise, robust localization algorithms are developed and analyzed by exploiting various forms of angular, temporal and spatial averages. The asymptotic convergence results have been established in the presence of noise and further the finite step performance results are developed.
\end{abstract}

Key words: Target Localization; .

\section{Introduction}

Over the last decades, target or source localization has received tremendous attention in the literature $[3,6-$ $8,10,13,16,14,19,22]$. The problem has applications in a wide range of areas, including wireless communications $[7,13,19,20]$, detection of radioactive materials $[4,17,18]$, airborne plume monitoring, surveillance and others.

Almost all localization algorithms assume availability of some form of signal propagation models emanating from the source $[6-8,10,14,19,20,22,13,16,18,17,4]$. [8] characterizes the bounds on localization accuracy if the signal strength model is available. [20] measures the received signal strength at sensors based on known signal propagation models and discusses ways to mitigate noise effects. [10] deals with the problem of source localization in diffusion processes, again assuming the signal strength model is available. [12] uses time of arrival or time difference of arrival and $[7,14,5]$ relies angle of arrival. These measurements provide distance or geometric information between sources and sensors. Therefore, triangulation techniques [4] or other methods can be used to localize sources. [6] discusses transmission power level. An-

\footnotetext{
^ The work was supported partially by NSF CNS-1239509. Email addresses: er-wei-bai@uiowa.edu (Er-wei Bai), liding@whu .edu.cn (Li Ding).
}

other way $[6,19]$ is to estimate the unknown parameters in the model and then to locate the source location based on the parameter estimates. These methods would not work if the structure of the model is unknown. There are also attempts $[4,11,13]$ to cast localization problems in a nonconvex optimization framework that also depends on the received signal propagation model.

All these approaches critically depend on the prior knowledge of the signal propagation model. However, in a number of applications such a description is unknown or only imprecisely known. While in $[5,19]$, methods for estimating these parameters are given, it is still potentially true that the shadowing model itself is inaccurate in certain environments. In radiation detection, signal models which are Poisson distributed involve several unknown parameters and in some cases even the structure of the signal model is unknown or in question $[17,18,23]$.

So an interesting and fundamental question is how to detect a source if signal propagation model is unknown. In contrast to approaches that rely on an available signal model, this work is based on one assumption that the signal strength received has a monotonic relationship with the distance between the source and sensor. The contribution of the paper is to theoretically demonstrate that the mere knowledge that a signal strength received decreases or increases with distance without any informa- 
tion of signal propagation model, is enough to locate a source exactly. Further in the presence of noise, robust algorithms are developed along with their convergence analyses. The paper is a continuation of our early work [2] where noise effect was neglected. This work only considers localization in a two dimensional space. However, all the results can be extended to any dimensional space with little modification.

The paper is organized as follows. Section 2 introduces the problems and presents some preliminary results. Robust localization algorithms by exploiting angular averages to reduce the effect of noise is presented and discussed in Section 3 along with a numerical simulation result. Convergence results have been established. Robust localization algorithms by exploiting temporal and spatial averages are presented and discussed in Sections 4 and 5. Section 6 provides some concluding remarks.

\section{Problem statement and preliminary results}

Let $y^{*}=\left(\begin{array}{c}y_{1}^{*} \\ y_{2}^{*}\end{array}\right) \in R^{2}$ be the unknown source location, $x(t)=\left(\begin{array}{l}x_{1}(t) \\ x_{2}(t)\end{array}\right) \in R^{2}$ the sensor location at time $t$ and $d^{*}(t)=\left\|x(t)-y^{*}\right\|$ the distance between the source and the sensor at time $t$. Here $t$ can be a continuous or a discrete variable. It is assumed that the received signal strength by the sensor at $x(t)$ is given by

$$
s(x(t))=g\left(d^{*}(t)\right)+v(x(t))
$$

where $v(\cdot)$ is a random noise variable and $g(\cdot)$ is a scalar smooth function with an unknown structure. The condition imposed on the unknown $g(\cdot)$ in this paper is the monotonicity.

\section{Assumption 2.1}

$$
g\left(d_{1}\right)>g\left(d_{2}\right) \text { if and only if } d_{1}<d_{2} .
$$

We would like to make some comments on the assumption.

- The most commonly used signal strength model in localization is in a form of

$$
s(x(t))=g\left(d^{*}(t)\right)=\frac{A}{d^{* 2}(t)} e^{-c d^{*}(t)}
$$

for some $A, c>0$ that belongs to this class. The difference of Assumption 2.1 with the existing literature is that no structural prior information on $g(\cdot)$ is imposed in the current paper.
- Assumption 2.1 does implicitly assume that the source strength is unknown but radially symmetric in the absence of noise. It may not be the case for some applications where various non-uniform fingerprints exist. How to relax Assumption 2.1 so the results can be extended to a non-radially symmetric case will be an interesting research topic.

- There is a huge difference between knowing the signal propagation model $g(\cdot)$ and not knowing. Suppose the signal propagation model is known, say as in (2). Based on the received signal strength $s(t)$ and values of $A, c$, the distance $d^{*}(t)=\left\|x(t)-y^{*}\right\|$ between the senor and the source can be calculated which can be used to locate the source for example by triangulation. The distance information is the key for most of work reported in the literature. Not knowing the model $g(\cdot)$, it is not possible to convert directly the received signal strength $s(t)$ into the distance information $d^{*}(t)=\left\|x(t)-y^{*}\right\|$. Thus, most of methods developed in the literature based on the received signal strength do not apply under Assumption 2.1.

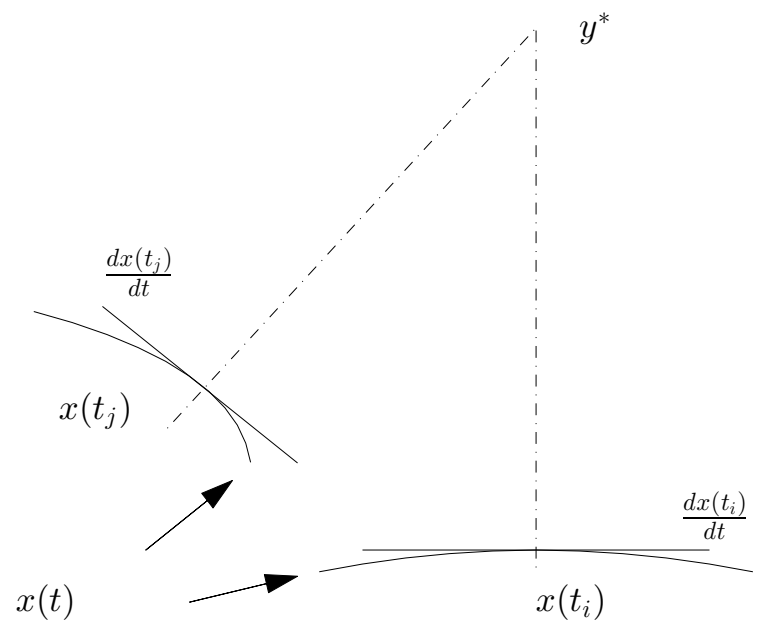

Fig. 1. Local maximum and the source location.

The goal of localization is to determine or estimate the unknown $y^{*}$ from sensor measurements $s(t)=s(x(t))$ and available sensor location $x(t)$. Localization without explicit information on $g(\cdot)$ has to exploit relative strength of the received signals. We exploit two approaches. First suppose in the absence of noise, $s(x(1))>s(x(2))$ at some sensor locations $x(1), x(2) \in$ $R^{2}$. Then, $d_{1}=\left\|x(1)-y^{*}\right\|<d_{2}=\left\|x(2)-y^{*}\right\|$. Now suppose a sensor is mobile which continuously records the strength $s(t)=s(x(t))$ as a function of time $t$. Assume further that the sensor path $x(t)$ is smooth and the derivative of $s(x(t))$ exists and is continuous,

$$
s^{\prime}(x(t))=\frac{d s}{d t}=\frac{d s}{d x} \frac{d x}{d t} .
$$

Then, $s^{\prime}(t)>0$ implies that the sensor is getting closer to the source $y^{*}$ and $s^{\prime}(t)<0$ implies that the sensor is 
moving away from the source. Further suppose $s(x(t))$ achieves a local (or the global) maximum at some sensor location $x\left(t_{i}\right)$ at time $t_{i}$ as shown in Figure 1. By the first order necessary condition $s^{\prime}\left(x\left(t_{i}\right)\right)=0$ and the fact that the distance $\left\|x\left(t_{i}\right)-y^{*}\right\|$ achieves the minimum in the neighborhood of $x\left(t_{i}\right)$ on the sensor path $x(t)$, the unknown but true $y^{*}$ has to be on the line that passes through $x\left(t_{i}\right)$ and is perpendicular to the tangent $\frac{d x\left(t_{i}\right)}{d t}$. Simply put $y^{*}$ is on the normal line at $x\left(t_{i}\right)$.

By summarizing the above analysis, we have the following result.

Theorem 2.1 Let $x(t)$ be the sensor path. Assume $\frac{d s}{d x}$ and $\frac{d x}{d t}$ exist and are continuous. Suppose $s(x(t))$ achieves two local maxima at $x\left(t_{i}\right)$ and $x\left(t_{j}\right)$ respectively. Further assume that the tangent lines $\frac{d x\left(t_{i}\right)}{d t}$ and $\frac{d x\left(t_{j}\right)}{d t}$ are linearly independent. Then in the absence of noise, the unknown source location $y^{*} \in R^{2}$ is uniquely determined at the intersection of two normal lines at $x\left(t_{i}\right)$ and $x\left(t_{j}\right)$ respectively.

The result is very useful as illustrated in Figure 1. In particular, let the area of interest be rectangular and the sensor path consist of two line segments in parallel to the rectangle as shown in Figure 2. Along the path, $s(x(t))$ achieves two and only two local maxima at some $x_{0}$ and $y_{0}$ respectively because of the monotonicity property of $s(x)$. The intersection of two normal lines at $x_{0}$ and $y_{0}$ is the source location $y^{*}$. A realistic problem is that local maxima and, consequently, $x_{0}$ and $y_{0}$ are hard to calculate reliably in the presence of noise.

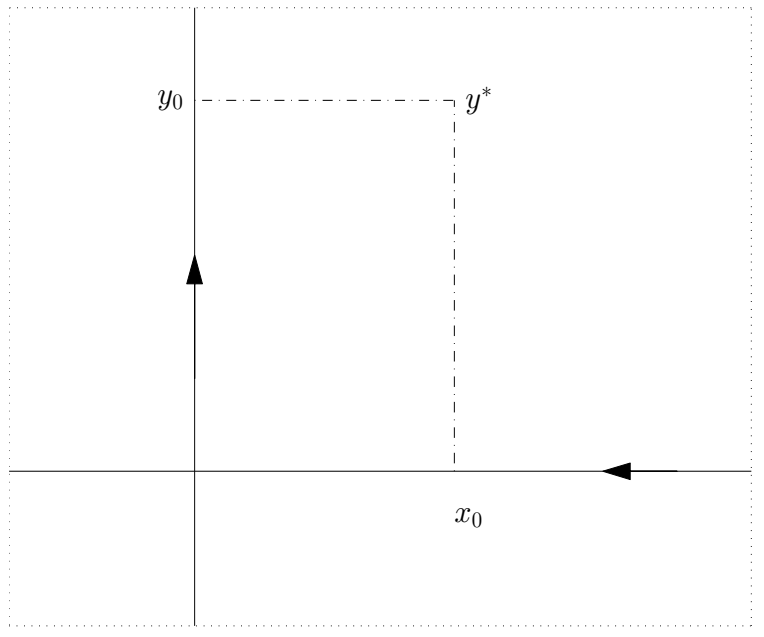

Fig. 2. Illustration of the result.

Another approach that we exploit in this paper is the relative direction of the unknown source with respect to the sensor location. Suppose in the absence of noise, a sensor were directional and were able to scan all the directions. Then, the direction that presents the strongest signal strength received by the sensor would be the direction where the unknown source lies. To make a sensor without explicit strength models become a directional sensor, we let the sensor make a circular movement centered at point $x(i) \in R^{2}$ with a radius $r>0$ resulting in a trajectory $z_{i}(\theta)=\left(\begin{array}{c}r \cos (\theta) \\ r \sin (\theta)\end{array}\right)+x(i), \theta \in[0,2 \pi)$, and at the same time, let the sensor continuously measure

$$
s\left(z_{i}(\theta)\right)=g_{i}(\theta)=g\left(\left\|z_{i}(\theta)-y^{*}\right\|\right)
$$

as shown in Figure 3.

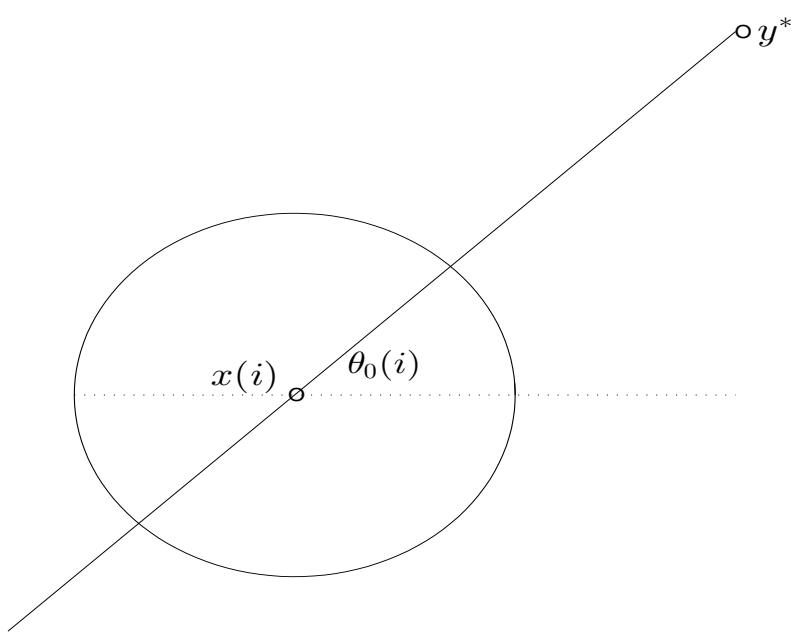

Fig. 3. Illustration of sensor circular movement.

We comment that the circular movement described above and the corresponding signals received (3) are sensor motion dependent and user defined. It is different from the work of $[7,14,5]$ reported in the literature which uses bearing information. In this paper, the only information available is the received signal strength $s(t)$ without knowledge of the signal propagation model $g(\cdot)$.

Now define a line connecting the center $x(i)$ to the unknown source location $y^{*}$. Let the line intersect the circle at $z_{i}\left(\theta_{0}(i)\right)$ and $z_{i}\left(\theta_{1}(i)\right)$ as in Figure 3 . In the absence of noise, since $g_{i}(\theta)=g\left(\left\|z_{i}(\theta)-y^{*}\right\|\right)$ is strictly monotonic in $\left\|z_{i}(\theta)-y^{*}\right\|$, we have

$$
z_{i}\left(\theta_{0}(i)\right)=\arg \min _{z_{i}(\theta)}\left\|z_{i}(\theta)-y^{*}\right\|
$$

or equivalently

$$
\theta_{0}(i)=\arg \max _{\theta} g_{i}(\theta)
$$

In summary, we have

Theorem 2.2 In the absence of noise for any given $x(i)$ and $r>0$, i.e., $s_{i}(\theta)=g_{i}(\theta)$, the angle $\theta_{0}(i)$ between the line that connects the center $x(i)$ and the source location $y^{*}$, and the horizontal axis is uniquely determined by (4) or equivalently the line connecting the center $x(i)$ to the source location $y^{*}$ is uniquely determined. 
Now if a sensor takes measurements around multiple circles at different centers $x(i)$ 's, the unknown $y^{*}$ is at the intersection of the lines connecting the source $y^{*}$ to the centers $x(i)$ 's, provided that these lines are not collinear. A practical problem is again how reliable the solution (4) is in the presence of noise.

Note in both approaches the exact value of $s(\cdot)$ is not important. What is important is if $s(x(t))$ achieves a local (or the global) maximum. So a key question is how to robustly determine if $s(x(t))$ achieves a local maximum or not in the presence of noise. We also comment that robust algorithm designs have to rely on the nature of noise. If noise is assumed to be bounded, the methods presented in $[1,24]$ can be useful. If a noise is assumed to be random, which is assumed in this paper, robust algorithms in some probability sense have to be developed to minimize the effect of random noise.

\section{Angular averages}



Fig. 4. Illustration of angular averages.

We first exploit the angular average approach. In the presence of noise, the received signal observed along the trajectory $z_{i}(\theta)$ centered at $x(i)$ is

$$
s_{i}(\theta)=g_{i}(\theta)+v_{i}(\theta), \quad \theta \in[0,2 \pi)
$$

where $v_{i}(\cdot)$ 's are assumed to be iid of zero mean and finite variance in both $i$ and $\theta$.

\subsection{Sampled values of $s(\cdot)$}

In practice, only a sampled version of $s_{i}(\theta)$ is available, where $\theta=k \delta \theta$ for some $\delta \theta$. To this end, divide $2 \pi$ by $(2 m+2)$ parts, $\delta \theta=\frac{2 \pi}{2 m+2}$ and set $k \delta \theta, k=0,1, \ldots, 2 m+$ 1 as shown in Figure 4 . Given a circle $z_{i}(\theta)$ centered at $x(i)$ with radius $r$, denote

$$
\theta_{0}(i)=k_{0}(i) \delta \theta, \text { for some } k_{0}(i) \in[0,2 m+2),
$$

and then the sampled version of $s(x(t))$ can be defined as

$$
s_{i}(k)=s_{i}(k \delta \theta)=g_{i}(k \delta \theta)+v_{i}(k \delta \theta)=g_{i}(k)+v_{i}(k) .
$$

\subsection{Robust estimates by the angular averages}

Let $\hat{k}_{0}(i)$ and $\hat{\theta}_{0}(i)$ be the estimates of $k_{0}(i)$ and $\theta_{0}(i)$ respectively defined by

$$
\begin{gathered}
\hat{k}_{0}(i)=\arg \min _{k} \frac{1}{m} \sum_{j=1}^{m}\left(s_{i}(k+j)-s_{i}(k-j)\right)^{2}, \\
k \pm j=k \pm j \bmod (2 m+2) . \\
\hat{\theta}_{0}(i)=\hat{k}_{0}(i) \delta \theta .
\end{gathered}
$$

Note unlike $\theta_{0}(i)$ in $(4)$, the estimates $\hat{k}_{0}(i)$ and $\hat{\theta}_{0}(i)$ are determined by minimizing the sum of the differences $\left(s_{i}(k+j)-s_{i}(k-j)\right)^{2}$. These estimates are much more robust than that of (4). Intuition is that, in the absence of noise

$$
s_{i}\left(k_{0}(i)+j\right)=s_{i}\left(k_{0}(i)-j\right)
$$

because $\left\|z_{i}\left(\left(k_{0}(i)+j\right) \delta \theta\right)-y^{*}\right\|=\left\|z_{i}\left(\left(k_{0}(i)-j\right) \delta \theta\right)-y^{*}\right\|$ which implies

$$
\frac{1}{m} \sum_{j=1}^{m}\left(s_{i}\left(k_{0}(i)+j\right)-s_{i}\left(k_{0}(i)-j\right)\right)^{2}=0,
$$

or $k_{0}(i)$ is the (one and only one) solution of the minimization (6). Now in the presence of noise,

$$
\begin{aligned}
& \frac{1}{m} \sum_{j=1}^{m}\left(s_{i}(k+j)-s_{i}(k-j)\right)^{2} \\
= & \frac{1}{m} \sum_{j=1}^{m}\left(g_{i}(k+j)+v_{i}(k+j)-g_{i}(k-j)-v_{i}(k-j)\right)^{2} \\
= & \frac{1}{m} \sum_{j=1}^{m}\left(g_{i}(k+j)-g_{i}(k-j)\right)^{2} \\
& +\frac{2}{m} \sum_{j=1}^{m}\left(g_{i}(k+j)-g_{i}(k-j)\right)\left(v_{i}(k+j)-v_{i}(k-j)\right) \\
& +\frac{1}{m} \sum_{j=1}^{m}\left(v_{i}(k+j)-v_{i}(k-j)\right)^{2} .
\end{aligned}
$$

It can be verified that in probability as $m \rightarrow \infty$, the second term goes to zero and the third term goes to a constant. Thus as $m \rightarrow \infty$,

$$
\arg \min _{k} \frac{1}{m} \sum_{j=1}^{m}\left(s_{i}(k+j)-s_{i}(k-j)\right)^{2}
$$




$$
\rightarrow \arg \min _{k} \frac{1}{m} \sum_{j=1}^{m}\left(g_{i}(k+j)-g_{i}(k-j)\right)^{2} .
$$

In other words, in probability as $m \rightarrow \infty$, the solutions of (6) and (7) satisfy

$$
\hat{k}_{0}(i) \rightarrow k_{0}(i), \hat{\theta}_{0}(i) \rightarrow \theta_{0}(i) .
$$

In summary, $\hat{k}_{0}(i)$ and $\hat{\theta}_{0}(i)$ can be written as

$$
\hat{k}_{0}(i)=k_{0}(i)+\Delta k_{0}(i), \hat{\theta}_{0}(i)=\theta_{0}(i)+\Delta \theta_{0}(i)
$$

where $\Delta k_{0}(i)$ and $\Delta \theta_{0}(i) \rightarrow 0$ as $m \rightarrow \infty$. The result is useful but asymptotical. A more interesting question is what can be said about $\Delta k_{0}(i)$ and $\Delta \theta_{0}(i)$ for any finite $m$. Note $2 m+2$ is the number of partitions of $\theta \in(0,2 \pi]$. To this end, we have

Theorem 3.1 For any $x(i)$ and $m>1, \Delta k_{0}(i)$ and $\Delta \theta_{0}(i)$ are random variables of zero mean and finite moments $E\left|\Delta \theta_{0}(i)\right|^{k}, k=1,2, \ldots, 6$, where $E$ is the expectation operator. Further, $\Delta k_{0}(i)$ and $\Delta k_{0}(j)$, and $\Delta \theta_{0}(j)$ and $\Delta \theta_{0}(j)$ are independent for $i \neq j$ provided that $x(i) \neq x(j)$.

Proof: We consider the case of $\Delta k_{0}$ only. Finite moments are clear since random variables are uniformly bounded by $\pi$. Independence is also clear because measurement noise at two circles centered at $x(i)$ and $x(j)$ are independent for $i \neq j$. To show zero mean, it suffices to show that the distribution is symmetric with respect to the origin. Let $\Delta k_{0}(i)=\hat{k}_{0}(i)-k_{0}(i)=k$ for some $k>0$.

$$
\begin{aligned}
& \operatorname{Prob}\left\{\Delta k_{0}(i)=k\right\} \\
= & \operatorname{Prob}\left\{\frac { 1 } { m } \sum _ { j = 1 } ^ { m } \left(g_{i}\left(k_{0}(i)+k+j\right)+v_{i}\left(k_{0}(i)+k+j\right)\right.\right. \\
& \left.-g_{i}\left(k_{0}(i)+k-j\right)-v_{i}\left(k_{0}(i)+k-j\right)\right)^{2} \\
\leq & \frac{1}{m} \sum_{j=1}^{m}\left(g_{i}(l+j)+v_{i}(l+j)-g_{i}(l-j)-v_{i}(l-j)\right)^{2}, \\
& \left.\forall l \neq k_{0}(i)+k\right\} .
\end{aligned}
$$

On the other hand,

$$
\begin{aligned}
& \operatorname{Prob}\left\{\Delta k_{0}(i)=-k\right\} \\
= & \operatorname{Prob}\left\{\frac { 1 } { m } \sum _ { j = 1 } ^ { m } \left(g_{i}\left(k_{0}(i)-k+j\right)+v_{i}\left(k_{0}(i)-k+j\right)\right.\right. \\
& \left.-g_{i}\left(k_{0}(i)-k-j\right)-v_{i}\left(k_{0}(i)-k-j\right)\right)^{2} \\
\leq & \frac{1}{m} \sum_{j=1}^{m}\left(g_{i}(l+j)+v_{i}(l+j)-g_{i}(l-j)-v_{i}(l-j)\right)^{2}, \\
& \left.\forall l \neq k_{0}(i)-k\right\},
\end{aligned}
$$

where

$g_{i}\left(k_{0}(i)-k+j\right)=g_{i}\left(k_{0}(i)-(k-j)\right)=g_{i}\left(k_{0}(i)+(k-j)\right)$, $g_{i}\left(k_{0}(i)-k-j\right)=g_{i}\left(k_{0}(i)-(k+j)\right)=g_{i}\left(k_{0}(i)+(k+j)\right)$, and $v_{i}(\cdot)$ 's are iid. Thus,

$$
\operatorname{Prob}\left\{\Delta k_{0}(i)=k\right\}=\operatorname{Prob}\left\{\Delta k_{0}(i)=-k\right\} .
$$

This shows that the distribution of $\Delta k_{0}(i)$ is symmetric with respect to the origin and hence the mean value is zero. This completes the proof.

We would like to comment that the above result is obtained assuming $\theta_{0}(i)=k_{0}(i) \delta \theta$ for some integer $k_{0}(i)$. In practice, for some choices of $\delta \theta, \theta_{0}(i)$ is not necessarily an integer multiple of $\delta \theta$ and consequently $\Delta \theta_{0}(i)$ is biased. The exact amount of bias depends on $m$. If $m$ is large, the bias is small even if bias is present. Also, the variance of $\Delta \theta_{0}(i)$ is a function of $m$. Both variance and bias go to zero as $m \rightarrow \infty$.

\subsection{Algorithm and convergence}

We now summarize the angular average algorithm.

The angular average algorithm: For given $n, m>1$ and $r>0$,

(1) Let the sensor path consist of $n$ circles $z_{i}(\theta)$ centered at $x(i)=\left(\begin{array}{l}x_{1}(i) \\ x_{2}(i)\end{array}\right), i=1, \ldots, n$ as in $(3)$

$$
s\left(z_{i}(\theta)\right)=g\left(\left\|z_{i}(\theta)-y^{*}\right\|\right)+v_{i}(\theta) .
$$

(2) For each $i$, let $\hat{\theta}_{0}(i)$ be defined as in (7). Observe that $\hat{\theta}_{0}(i)$ can be written as

$\hat{\theta}_{0}(i)=\theta_{0}(i)+\Delta \theta_{0}(i)=\arctan \frac{y_{2}^{*}-x_{2}(i)}{y_{1}^{*}-x_{1}(i)}+\Delta \theta_{0}(i)$,

where $\Delta \theta_{0}(i)$ 's are independent, zero mean and finite variance $\sigma_{i}^{2}$ 's. $\Delta \theta_{0}(i)$ 's are not necessarily identically distributed.

(3) Based on $\hat{\theta}_{0}(i)$ 's, $i=1,2, \ldots, n$, an estimate $\hat{y}=$ $\left(\begin{array}{l}\hat{y}_{1} \\ \hat{y}_{2}\end{array}\right)$ of $y^{*}=\left(\begin{array}{l}y_{1}^{*} \\ y_{2}^{*}\end{array}\right)$ can be defined

$$
\hat{y}=\arg \min _{\hat{y}=\left(\begin{array}{c}
\hat{y}_{1} \\
\hat{y}_{2}
\end{array}\right)} \frac{1}{n} \sum_{i=1}^{n}\left(\hat{\theta}_{0}(i)-\arctan \frac{\hat{y}_{2}-x_{2}(i)}{\hat{y}_{1}-x_{1}(i)}\right)^{2} .
$$

Theorem 3.2 Assume $\tan \hat{\theta}_{0}(i)$ and $\tan \theta_{0}(i)$ are uniformly bounded, i.e., there exists a constant $c>0$ such 
that $\left|\tan \hat{\theta}_{0}(i)\right|,\left|\tan \theta_{0}(i)\right| \leq c, i, 1, \ldots, n$. Then, the estimate $\hat{y}$ of (10) satisfies in distribution as $n \rightarrow \infty$,

$$
\hat{y} \rightarrow y^{*}
$$

provided that

$$
\begin{array}{r}
\lim _{n \rightarrow \infty}\left(\frac{1}{n} \sum_{i=1}^{n} \tan ^{2} \theta_{0}(i)-\left(\frac{1}{n} \sum_{i=1}^{n} \tan \theta_{0}(i)\right)^{2}\right) \\
=\lim _{n \rightarrow \infty} \frac{1}{2 n^{2}} \sum_{i=1}^{n} \sum_{j=1}^{n}\left(\tan \theta_{0}(i)-\tan \theta_{0}(j)\right)^{2}>0 .
\end{array}
$$

Proof:

$$
\begin{aligned}
& \frac{1}{n} \sum_{i=1}^{n}\left(\theta_{0}(i)+\Delta \theta_{0}(i)-\arctan \frac{\hat{y}_{2}-x_{2}(i)}{\hat{y}_{1}-x_{1}(i)}\right)^{2} \\
= & \frac{1}{n} \sum_{i=1}^{n}\left(\theta_{0}(i)-\arctan \frac{\hat{y}_{2}-x_{2}(i)}{\hat{y}_{1}-x_{1}(i)}\right)^{2} \\
& +\frac{2}{n} \sum_{i=1}^{n}\left(\theta_{0}(i)-\arctan \frac{\hat{y}_{2}-x_{2}(i)}{\hat{y}_{1}-x_{1}(i)}\right) \Delta \theta_{0}(i) \\
& +\frac{1}{n} \sum_{i=1}^{n} \sigma_{i}^{2}+\frac{1}{n} \sum_{i=1}^{n}\left(\Delta^{2} \theta_{0}(i)-\sigma_{i}^{2}\right),
\end{aligned}
$$

where $\sigma_{i}^{2}$ is the variance of $\Delta \theta_{0}(i)$. The second term goes to zero in probability as $n \rightarrow \infty$ since $\Delta \theta_{0}(i)$ 's are independent, uniformly bounded and zero mean. The third term is independent of $\hat{y}$ and can be removed from the optimization (10). From Theorem 3.1, $E\left(\Delta^{2} \theta_{0}(i)-\sigma_{i}^{2}\right)^{2}=$ $\mu_{i} \leq \mu<\infty$ for some constant $\mu$. Now consider the last term. By the Berry-Essen Theorem [9], the distribution of the random variable $\frac{\sum_{i=1}^{n}\left(\Delta^{2} \theta_{0}(i)-\sigma_{i}^{2}\right)}{\sqrt{\mu_{1}+\ldots+\mu_{n}}}$ converges in distribution to a Gaussian of zero mean and unit variance,

$$
\begin{aligned}
& \frac{\sum_{i=1}^{n}\left(\Delta^{2} \theta_{0}(i)-\sigma_{i}^{2}\right)}{\sqrt{\mu_{1}+\ldots+\mu_{n}}} \\
= & \sqrt{n} \sqrt{\frac{n}{\mu_{1}+\ldots+\mu_{n}}} \frac{\sum_{i=1}^{n}\left(\Delta^{2} \theta_{0}(i)-\sigma_{i}^{2}\right)}{n} \sim N(0,1) .
\end{aligned}
$$

Further,

$$
\sqrt{\frac{n}{\mu_{1}+\ldots+\mu_{n}}} \geq \sqrt{\frac{n}{n \mu}}=\frac{1}{\sqrt{\mu}} .
$$

This implies, for some $0 \leq \alpha(n) \leq \mu<\infty$,

$$
\frac{\sum_{i=1}^{n}\left(\Delta^{2} \theta_{0}(i)-\sigma_{i}^{2}\right)}{n} \sim N\left(0, \frac{\alpha(n)}{n}\right) .
$$

Therefore in distribution

$$
\begin{aligned}
& \frac{1}{n} \sum_{i=1}^{n}\left(\theta_{0}(i)+\Delta \theta_{0}(i)-\arctan \frac{\hat{y}_{2}-x_{2}(i)}{\hat{y}_{1}-x_{1}(i)}\right)^{2} \\
\rightarrow & \frac{1}{n} \sum_{i=1}^{n}\left(\theta_{0}(i)-\arctan \frac{\hat{y}_{2}-x_{2}(i)}{\hat{y}_{1}-x_{1}(i)}\right)^{2} .
\end{aligned}
$$

Now observe under the assumptions,

$$
\frac{1}{n} \sum_{i=1}^{n}\left(\theta_{0}(i)-\arctan \frac{y_{2}^{*}-x_{2}(i)}{y_{1}^{*}-x_{1}(i)}\right)^{2}=0
$$

and

$$
\theta_{0}(i)=\arctan \frac{\hat{y}_{2}-x_{2}(i)}{\hat{y}_{1}-x_{1}(i)} \Leftrightarrow \tan \theta_{0}(i)=\frac{\hat{y}_{2}-x_{2}(i)}{\hat{y}_{1}-x_{1}(i)} .
$$

The question is if (13) has multiple solutions of $\hat{y}$. To this end,

$$
\begin{aligned}
& \frac{1}{n} \sum_{i=1}^{n}\left(\theta_{0}(i)-\arctan \frac{\hat{y}_{2}-x_{2}(i)}{\hat{y}_{1}-x_{1}(i)}\right)^{2}=0 \\
& \Leftrightarrow \frac{1}{n} \sum_{i=1}^{n}\left(\tan \theta_{0}(i)-\frac{\hat{y}_{2}-x_{2}(i)}{\hat{y}_{1}-x_{1}(i)}\right)^{2}=0 \\
& \Leftrightarrow \frac{1}{n} \sum_{i=1}^{n}\left[\left(\tan \theta_{0}(i),-1\right)\left(\begin{array}{l}
\hat{y}_{1} \\
\hat{y}_{2}
\end{array}\right)\right. \\
& \left.-\left(\tan \theta_{0}(i) x_{1}(i)-x_{2}(i)\right)\right]^{2}=0 \\
& \Leftrightarrow \| \frac{1}{\sqrt{n}}\left(\begin{array}{cc}
\tan \theta_{0}(1) & -1 \\
\vdots & \vdots \\
\tan \theta_{0}(n) & -1
\end{array}\right)\left(\begin{array}{l}
\hat{y}_{1} \\
\hat{y}_{2}
\end{array}\right) \\
& -\frac{1}{\sqrt{n}}\left(\begin{array}{c}
\tan \theta_{0}(1) x_{1}(1)-x_{2}(1) \\
\vdots \\
\tan \theta_{0}(1) x_{1}(n)-x_{2}(n)
\end{array}\right) \|^{2}=0 .
\end{aligned}
$$

The solution is unique if and only if

$$
\begin{gathered}
0<\frac{1}{n}\left(\begin{array}{ccc}
\tan \theta_{0}(1) & \cdots & \tan \theta_{0}(n) \\
-1 & \cdots & -1
\end{array}\right)\left(\begin{array}{cc}
\tan \theta_{0}(1) & -1 \\
\vdots & \vdots \\
\tan \theta_{0}(n) & -1
\end{array}\right) \\
=\left(\begin{array}{cc}
\frac{1}{n} \sum_{i=1}^{n} \tan ^{2} \theta_{0}(i) & -\frac{1}{n} \sum_{i=1}^{n} \tan \theta_{0}(i) \\
-\frac{1}{n} \sum_{i=1}^{n} \tan \theta_{0}(i) & -1
\end{array}\right) \\
\Leftrightarrow \frac{1}{n} \sum_{i=1}^{n} \tan ^{2} \theta_{0}(i)-\left(\frac{1}{n} \sum_{i=1}^{n} \tan \theta_{0}(i)\right)^{2}
\end{gathered}
$$




$$
=\frac{1}{2 n^{2}} \sum_{i=1}^{n} \sum_{j=1}^{n}\left(\tan \theta_{0}(i)-\tan \theta_{0}(j)\right)^{2}>0 .
$$

This finishes the proof.

Now the question is when the assumption (11) is satisfied.

Corollary 3.1 (1) Let $\tan \theta_{0}(i)$ 's be iid of variance $\sigma_{\theta}^{2}>0$. Then in probability as $n \rightarrow \infty$,

$$
\begin{aligned}
& \frac{1}{n} \sum_{i=1}^{n} \tan ^{2} \theta_{0}(i)-\left(\frac{1}{n} \sum_{i=1}^{n} \tan \theta_{0}(i)\right)^{2} \\
\rightarrow & E\left(\tan ^{2} \theta_{0}(i)\right)-\left(E \tan \theta_{0}(i)\right)^{2}=\sigma_{\theta}^{2}>0 .
\end{aligned}
$$

(2) Consider a sequence $\tan \theta_{0}(i), i=1, \ldots, n$ and a subsequence $\tan \theta_{0}\left(i_{k}\right), k=1, \ldots, j$, where $j \geq n / l$ for some positive integer $l>0$. Suppose $\tan \theta_{0}(i)$ 's are iid of variance $\sigma_{\theta}^{2}>0$. Then,

$$
\begin{aligned}
& \frac{1}{n} \sum_{i=1}^{n} \tan ^{2} \theta_{0}(i)-\left(\frac{1}{n} \sum_{i=1}^{n} \tan \theta_{0}(i)\right)^{2} \\
\rightarrow & E\left(\tan ^{2} \theta_{0}(i)\right)-\left(E \tan \theta_{0}(i)\right)^{2}>0 .
\end{aligned}
$$

Proof: We only show the claim 2. Clearly as $n \rightarrow \infty$, $j \rightarrow \infty$. Then

$$
\begin{aligned}
& \frac{1}{2 n^{2}} \sum_{i=1}^{n} \sum_{m=1}^{n}\left(\tan \theta_{0}(i)-\tan \theta_{0}(m)\right)^{2} \\
\geq & \frac{1}{2 n^{2}} \sum_{i=1}^{j} \sum_{m=1}^{j}\left(\tan \theta_{0}(i)-\tan \theta_{0}(m)\right)^{2} \rightarrow \frac{1}{l^{2}} \sigma_{\theta}^{2}>0 .
\end{aligned}
$$

This completes the proof.

A rough translation of the result is that if the centers $x(i)$ 's are randomly assigned, the estimate of (10) converges to the unknown $y^{*}$ asymptotically.

\subsection{A numerical example}

To illustrate the performance of the proposed algorithm, we consider an example. Let the area be a square with the side length $l=200$ (units). The unknown source is at $y^{*}=\left(\begin{array}{c}110 \\ 95\end{array}\right)$ and $s_{i}(\theta)=g_{i}(\theta)+v_{i}(\theta)$ where $v_{i}(\cdot)$ 's are iid Gaussian of zero mean and $\mathrm{std}=0.1$, and $g=\frac{1200}{d_{i}^{2}} e^{-0.006 d_{i}}$ where $d_{i}=\left\|z_{i}(\theta)-y^{*}\right\|$. No knowledge of $g_{i}(\cdot)^{i}$ was used in simulations. For simulations, $m=$ 61 and $r=5=d_{\max }$ and the centers of the sensor are randomly and uniformly assigned in the area. Table 1 shows the estimates, which are the averages of 100

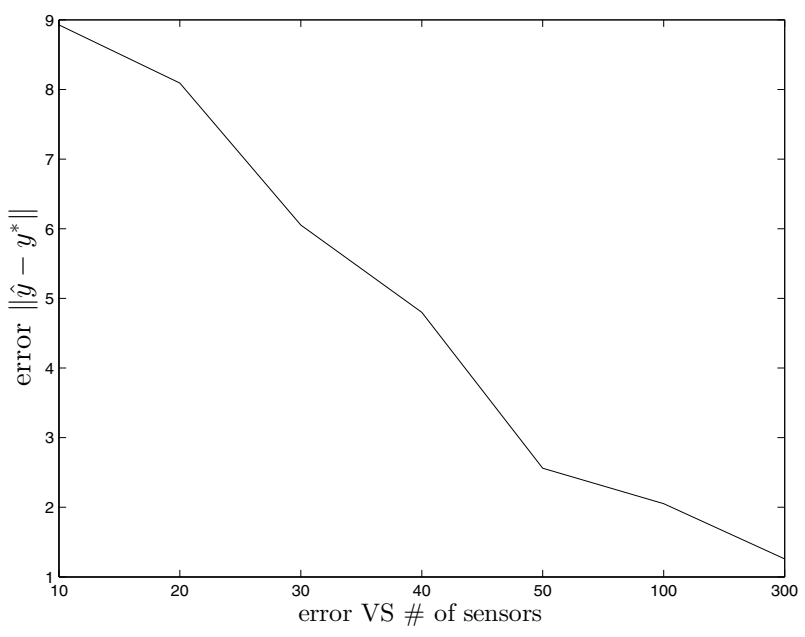

Fig. 5. Estimation error vs the number of sensors.

\begin{tabular}{|c|c|}
\hline$y^{*}=\left(\begin{array}{c}110 \\
95\end{array}\right)$, noise std $=0.1, r=d_{\text {max }}=5, m=61$ \\
\hline \# of sensors $x(i)$ 's & $\left(\begin{array}{c}112.21 \\
103.65\end{array}\right)\left(\begin{array}{l}1982.3 \\
2712.2\end{array}\right)$ \\
\hline 10 & $\left(\begin{array}{c}102.4 \\
92.22\end{array}\right)\left(\begin{array}{l}996.59 \\
1900.6\end{array}\right)$ \\
\hline 20 & $\left(\begin{array}{c}104.29 \\
97.01\end{array}\right)\left(\begin{array}{l}784.97 \\
1257.7\end{array}\right)$ \\
\hline 30 & $\left(\begin{array}{c}105.91 \\
97.51\end{array}\right)\left(\begin{array}{l}706.48 \\
1927.5\end{array}\right)$ \\
\hline 40 & $\left(\begin{array}{c}109.22 \\
92.56\end{array}\right)\left(\begin{array}{l}706.54 \\
1816.5\end{array}\right)$ \\
\hline 100 & $\left(\begin{array}{c}108.51 \\
93.59\end{array}\right)\left(\begin{array}{l}152.15 \\
269.86\end{array}\right)$ \\
\hline 300 & $\left(\begin{array}{c}108.76 \\
94.78\end{array}\right)\left(\begin{array}{l}10.69 \\
5.68\end{array}\right)$ \\
\hline 1
\end{tabular}

Table 1

The estimates $\hat{y}$ 's and their variances in the first and second parentheses respectively.

Monte Carlo runs, and the variance of the estimates in the second parenthesis based on the 100 runs. Figure 5 provides the estimation errors graphically.

\section{Temporal averages}

The idea of the paper is that suppose $s(x(t))$ achieves a local maximum at some sensor location $x\left(t_{i}\right)$. Then, the unknown $y^{*}$ has to be on the normal line of the sensor trajectory $x(t)$ at $x\left(t_{i}\right)$. Two sensor motion trajectories 
are proposed, the circular or angular motion approach in Figure 3 and the line path motion approach in Figure 2.

In the previous section, the robust estimates are proposed and studied based on the angular average approach. In this section, we develop robust estimates based on line motion paths. The idea is an average of multiple samples to average out the effect of noise. To focus on the idea and avoid all unnecessary complications, let the area considered be a rectangle in parallel to the axes and the low left corner of the rectangle sits on the origin. Further let the sensor path be two lines in parallel to the axes as shown in Figure 2. These assumptions are for simplicity alone and the results apply to other shapes of areas and paths equally.

\subsection{Robust estimates based on the line paths}

In the absence of noise, the local maxima $x_{0}$ and $y_{0}$ as shown in Figure 2 can be easily obtained by the line paths. To develop robust estimates in the presence of noise, let us consider the horizontal sensor path first in which the $y$-coordinate is fixed at some $\bar{y}$. The received signal along this path is strictly increasing until $x(t)=x_{0}$ and then is strictly decreasing at least in the absence of noise as shown in Figure 2. Let the length of the rectangle in the horizontal direction be $l$ (units) and $\Delta x=l / n$ be the sampled step. Then the received signal along the horizontal path can be written as

$$
\begin{aligned}
& s_{h}\left(k-k_{x}\right)=s_{h}\left(\left(k-k_{x}\right) \Delta x\right) \\
= & g\left(\left\|\left(\begin{array}{c}
k \Delta x \\
\bar{y}
\end{array}\right)-y^{*}\right\|\right)+v(k \Delta x), k=0,1, \ldots, n
\end{aligned}
$$

which is a sampled version of $s(x)$ along the horizontal sensor path. $v(\cdot)$ 's represent iid random noise of mean $\mu$ and finite variance $\sigma^{2}$. For simplicity, we assume $\mu=0$. Let $g_{h}(k)=g\left(\left\|\left(\begin{array}{c}k \Delta x \\ \bar{y}\end{array}\right)-y^{*}\right\|\right)$ and $x_{0}=k_{x} \Delta x$ for some unknown $k_{x}$ at which $g_{h}(\cdot)$ achieves a local maximum. Obviously, $g_{h}(k)=g\left(\left\|\left(\begin{array}{c}k \Delta x \\ \bar{y}\end{array}\right)-y^{*}\right\|\right)$ is positive, symmetric with respect to $k_{x}$, and strictly decreasing in $\left\|k-k_{x}\right\|$. The goal is to determine

$$
k_{x}=\arg \min _{k} g\left(\left\|\left(\begin{array}{c}
k \Delta x \\
\bar{y}
\end{array}\right)-y^{*}\right\|\right)
$$

and consequently $x_{0}=k_{x} \Delta x$ from $s_{h}\left(k-k_{x}\right)$, a corrupted version of $g(\cdot)$ by noise $v(\cdot)$. In the absence of noise, $k_{x}$ can be easily found by solving $\arg \max _{k} s_{h}(k-$ $\left.k_{x}\right)$. In the presence of noise, a direct way to find $k_{x}$ is by solving

$$
\hat{k}_{x}=\arg \max _{k} s_{h}\left(k-k_{x}\right)=\arg \max _{k}\left\{g_{h}(k)+v(k)\right\} .
$$

The solution however is sensitive to noise. How to make $\hat{k}_{x}$ close to the true but unknown $k_{x}$ is the goal. Suppose the signal $s_{h}\left(k-k_{x}\right)$ are measured multiple times, say $L$ times that result in

$$
s_{h j}\left(k-k_{x}\right), j=1, \ldots, L, k=0,1, \ldots, n
$$

which can be obtained either by the sensor moving along the horizontal path repeatedly $L$ times or stopping at each point and taking the measurements $L$ times. Now, define

$$
s_{h}^{L}\left(k-k_{x}\right)=\frac{1}{L} \sum_{j=1}^{L} s_{h j}\left(k-k_{x}\right)
$$

and consider

$$
\begin{aligned}
\hat{k}_{x} & =\arg \max _{k} s_{h}^{L}\left(k-k_{x}\right) \\
& =\arg \max _{k} \frac{1}{L} \sum_{j=1}^{L} s_{h j}\left(k-k_{x}\right) \\
& =\arg \max _{k}\left\{g_{h}(k)+\bar{v}_{L}(k)\right\}
\end{aligned}
$$

where $\bar{v}_{L}(k)=\frac{1}{L} \sum_{j=1}^{L} v_{j}(k)$ 's are iid of zero mean and variance $\sigma^{2} / L$. Consequently, we have

Theorem 4.1 The estimate $\hat{k}_{x}$ (14) of $k_{x}$ converges to $k_{x}$ in probability as $L \rightarrow \infty$.

\subsection{Quantification of errors for a given $L$}

The above result tells us that if measurements can be repeated $L$ times, the variance of the estimate is reduced by a factor of $L$. Further $L \rightarrow \infty$ implies that the estimation error $\rightarrow 0$. In practice, however, $L$ is always finite and a more useful result would be, for a given $L$, a quantification of the error $\left|k_{x}-\hat{k}_{x}\right|$ between the true $k_{x}$ and its estimate $\hat{k}_{x}$ in some probabilistic sense. To this end, let $m>0$ be the given error bound on $k_{x}$ or equivalently $m \Delta x$ the error bound on $\left|\hat{x}_{0}-x_{0}\right|$ where $\hat{x}_{0}=\hat{k}_{x} \Delta x$ and $\Delta x=l / n$ is the sampled size. What we are interested is the probability of $\left|\hat{k}_{x}-k_{x}\right| \leq m$,

$$
\begin{aligned}
\rho_{x} & =\operatorname{Prob}\left\{\max _{k \in\left[k_{x}-m, k_{x}+m\right]} s_{h}^{L}\left(k-k_{x}\right)\right. \\
& \left.>\max _{k \notin\left[k_{x}-m, k_{x}+m\right]} s_{h}^{L}\left(k-k_{x}\right)\right\}
\end{aligned}
$$

for a given pair $(L, m)$. This probability $\rho_{x}$, together with a similar probability $\rho_{y}$ on the vertical path, characterize the estimation accuracy. Intuitively as shown in Figure 6, the estimate $\hat{y}=\left(\begin{array}{c}\hat{y}_{1} \\ \hat{y}_{2}\end{array}\right)$ of $y^{*}$ is at the intersection of the 
normal lines at $\hat{y}_{1}=\hat{x}_{0}=\hat{k}_{x} \Delta x$ and $\hat{y}_{2}=\hat{y}_{0}=\hat{k}_{y} \Delta y$. Further, the probability that the true but unknown $y^{*}$ lies in a box cornered by

$$
\left[\left(\hat{k}_{x}-m\right) \Delta x,\left(\hat{k}_{x}+m\right) \Delta x\right] \times\left[\left(\hat{k}_{y}-m\right) \Delta y,\left(\hat{k}_{x}+m\right) \Delta y\right]
$$

is $\rho_{x} \rho_{y}$. Here the same error bound $m$ is used for both horizontal and vertical directions. Localization is successful if the probability $\rho_{x} \rho_{y}$ is high and the error bounds $m \Delta x$ and $m \Delta y$ are acceptable.

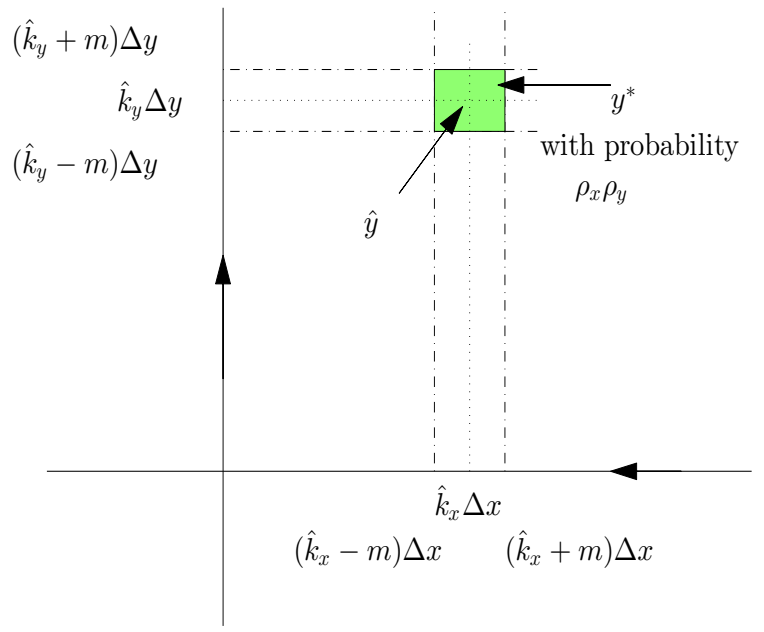

Fig. 6. Error bounds $\left|\hat{k}_{x}-k_{x}\right|,\left|\hat{k}_{y}-k_{y}\right| \leq m$.

The problem is that calculation of (15) is non-trivial. In fact, the exact calculation is impossible since it involves the unknown function $g(\cdot)$. Therefore, a reasonable alternative is to find a good estimate of the probability (15), at least for some large $n$.

Let $n_{1}=2 m+1$ which is the number of points in $k \in$ $\left[k_{x}-m, k_{x}+m\right]$ and $n_{2}=n-2 m-1$, the number of points in $k \notin\left[k_{x}-m, k_{x}+m\right]$ and let

$$
\begin{aligned}
u_{m} & =\max _{k \in\left[k_{x}-m, k_{x}+m\right]} s_{h}^{L}\left(k-k_{x}\right), \\
w_{m} & =\max _{k \notin\left[k_{x}-m, k_{x}+m\right]} s_{h}^{L}\left(k-k_{x}\right) .
\end{aligned}
$$

Let $p_{1}(u)$ and $p_{2}(w)$ be the (unknown) probabilistic density functions of random variables $u_{m}$ and $w_{m}$ respectively. The probability (15) that we want to calculate becomes

$$
\begin{gathered}
\rho_{x}=\operatorname{Prob}\left\{\left|\hat{k}_{x}-k_{x}\right| \leq m\right\}=\operatorname{Prob}\left\{u_{m}>w_{m}\right\} \\
=\iint_{u>w} p_{1}(u) p_{2}(w) d u d w
\end{gathered}
$$

The last equality follows from the fact that $u_{m}$ and $w_{m}$ are independent and the joint density is the product of individual ones. Clearly if $p_{1}(u)$ and $p_{2}(w)$ are available, the probability of (16) can be computed at least numerically since it is a two dimensional integration. A difficulty again is how to estimate $p_{1}(u)$ and $p_{2}(w)$. To this end, a number of obstacles have to be dealt with.

Lemma 4.1 Let $\nu_{1}, \nu_{2}, \ldots, \nu_{n}$ be iid Gaussian random variables of mean $\mu$ and variance $\sigma^{2} / L$ and

$$
z_{n}=\max \left\{\nu_{1}, \nu_{2}, \ldots, \nu_{n}\right\}
$$

Then, the cumulative distribution $F(z)$ and the density function $p(z)$ of $z_{n}$ converge to, as $n \rightarrow \infty$,

$$
\begin{aligned}
& F(z)=\operatorname{Prob}\left\{z_{n}<z\right\} \rightarrow \exp \left(-\exp \left(-\frac{\frac{z-\mu}{\sigma / \sqrt{L}}-a_{n}}{b_{n}}\right)\right) \\
& p(z)=\frac{d F}{d z} \rightarrow \\
& n \exp \left(-\exp \left(-\frac{\frac{z-\mu}{\sigma / \sqrt{L}}-a_{n-1}}{b_{n-1}}\right)\right) \frac{1}{\sqrt{2 \pi \sigma^{2} / L}} e^{-\frac{(z-\mu)^{2}}{2 \sigma^{2} / L}}
\end{aligned}
$$

where

$$
a_{n}=(2 \ln n)^{1 / 2}-\frac{\frac{1}{2}(\ln (\ln n))+\ln (4 \pi)}{(2 \ln n)^{1 / 2}}
$$

$$
b_{n}=2(\ln n)^{-1 / 2}
$$

Proof:

$$
\begin{aligned}
F(z) & =\operatorname{Prob}\left\{z_{n} \leq z\right\} \\
& =\operatorname{Prob}\left\{\nu_{1} \leq z, \ldots, \nu_{n} \leq z\right\} \\
& =\left(\int_{-\infty}^{z} \frac{1}{\sqrt{2 \pi \sigma^{2} / L}} e^{-\frac{(v-\mu)^{2}}{2 \sigma^{2} / L}} d v\right)^{n} \\
& =\left(\int_{-\infty}^{\frac{z-\mu}{\sigma / \sqrt{L}}} \frac{1}{\sqrt{2 \pi}} e^{-\frac{v^{2}}{2}} d v\right)^{n},
\end{aligned}
$$

and the density is

$$
\begin{aligned}
p(z) & =\frac{d F(z)}{d z} \\
& =n\left(\int_{-\infty}^{\frac{z-\mu}{\sigma / \sqrt{L}}} \frac{1}{\sqrt{2 \pi}} e^{-\frac{v^{2}}{2}} d v\right)^{n-1} \frac{1}{\sqrt{2 \pi \sigma^{2} / L}} e^{-\frac{(z-\mu)^{2}}{2 \sigma^{2} / L}} .
\end{aligned}
$$

By the asymptotic theory of extreme order statistics [15],

$\lim _{n \rightarrow \infty}\left(\int_{-\infty}^{z} \frac{1}{\sqrt{2 \pi}} e^{-\frac{v^{2}}{2}} d v\right)^{n}=\lim _{n \rightarrow \infty} \exp \left(-\exp \left(-\frac{z-a_{n}}{b_{n}}\right)\right)$. 
Therefore the cumulative distribution $F(z)$ follows. Further,

$$
\begin{aligned}
& p(z)=\frac{d F}{d z} \\
& \rightarrow n \exp \left(-\exp \left(-\frac{\frac{z-\mu}{\sigma / \sqrt{L}}-a_{n-1}}{b_{n-1}}\right)\right) \frac{1}{\sqrt{2 \pi \sigma^{2} / L}} e^{-\frac{(z-\mu)^{2}}{2 \sigma^{2} / L}} .
\end{aligned}
$$

This completes the proof.

We comment that the Gaussian assumption on the noise was used in deriving the limit distribution (18). This is not a restriction. Such limit distributions are available for most commonly used distributions. For instance, let the noise distribution be uniform in $(0,1)$, i.e.,

$$
F(z)=\left\{\begin{array}{l}
0, z<0 \\
z, 0 \leq z \leq 1 \\
1, z>1
\end{array}\right.
$$

Then from [15],

$$
\lim _{n \rightarrow \infty} \operatorname{Prob}\left\{z_{n}<1+\frac{1}{n} z\right\}=\left\{\begin{array}{l}
e^{z}, z<0, \\
1, \quad z \geq 0 .
\end{array}\right.
$$

In fact by the Fisher-Tippett-Gnedenko Theorem [15], the exact form of the distribution is not needed to find the limit distribution. There are only three types of limit distributions depending on the tails of the distribution, a light tail, a heavy tail (including polynomial decay) and an exponential tail [15].

The second obstacle is that (17) is the limit density of iid Gaussian with the same mean value and in our case, the mean value of $s_{h}\left(k-k_{x}\right)$ is $g_{h}(k)$ which is a function of $k$. To apply (17) we may assume the mean value is the same for $k \in\left[k_{x}-m, k_{x}+m\right]$ and for $k \notin\left[k_{x}-m, k_{x}+m\right]$. To be precise, let the mean value of $g_{h}(k)$ be $s 1=g_{h}\left(k_{x}+m\right)$ for $k \in\left[k_{x}-m, k_{x}+m\right]$ which is a lower bound on $g_{h}(k)$ for $k \in\left[k_{x}-m, k_{x}+m\right]$ that provides a lower bound on the probability density, and the mean value be $s 2=$ $\left(g_{h}\left(k_{x}+m\right)-g_{h}(n)\right) / 2+g_{h}(n)$ for $k \notin\left[k_{x}-m, k_{x}+m\right]$ that is the value of $g_{h}$ at the middle point of the interval. Then, approximations of $p_{1}(u)$ and $p_{2}(w)$ are obtained,

$$
\begin{aligned}
& \hat{p}_{1}(u)=n_{1}\left(\int_{-\infty}^{\frac{u-s 1}{\sigma / \sqrt{L}}} \frac{1}{\sqrt{2 \pi}} e^{-\frac{v^{2}}{2}} d v\right)^{n_{1}-1} \frac{1}{\sqrt{2 \pi \sigma^{2} / L}} e^{-\frac{(u-s 1)^{2}}{2 \sigma^{2} / L}} \\
& \rightarrow n_{1} \exp \left(-\exp \left(-\frac{\frac{u-s 1}{\sigma / \sqrt{L}}-a_{n_{1}-1}}{b_{n_{1}-1}}\right)\right) \frac{1}{\sqrt{2 \pi \sigma^{2} / L}} e^{-\frac{(u-s 1)^{2}}{2 \sigma^{2} / L}}
\end{aligned}
$$

$$
\begin{aligned}
& \hat{p}_{2}(w)=n_{2}\left(\int_{-\infty}^{\frac{w-s 2}{\sigma / \sqrt{L}}} \frac{1}{\sqrt{2 \pi}} e^{-\frac{v^{2}}{2}} d v\right)^{n_{2}-1} \\
& \cdot \frac{1}{\sqrt{2 \pi \sigma^{2} / L}} e^{-\frac{(w-s 2)^{2}}{2 \sigma^{2} / L}} \\
& \rightarrow n_{2} \exp \left(-\exp \left(-\frac{\frac{w-s 2}{\sigma / \sqrt{L}}-a_{n_{2}-1}}{b_{n_{2}-1}}\right)\right) \frac{1}{\sqrt{2 \pi \sigma^{2} / L}} e^{-\frac{(w-s 2)^{2}}{2 \sigma^{2} / L}} .
\end{aligned}
$$

The last obstacle is that the values of $g_{h}\left(k_{x}+m\right)$ and $g_{h}(n)$ are unknown. On the other hand, $\hat{k}_{x}$ of $(14)$ is available and so are the estimates $\widehat{s 1}=s_{h}^{L}\left(\hat{k}_{x}+m\right)$ and $\widehat{s 2}=\left(s_{h}^{L}\left(\hat{k}_{x}+m\right)-s_{h}^{L}(n)\right) / 2+s_{h}^{L}(n)$. We are now in a position to state the algorithm to estimate $y^{*}$ and the probability of (16).

\section{Algorithm for the estimates of the unknown source location $y^{*}$ and the probability (16) of the estimates bounds:}

(1) Let the unknown $y^{*}$ lie in a rectangle of $\left[0, l_{h}\right] \times$ $\left[0, l_{v}\right]\left(\right.$ units $\left.^{2}\right)$, where the subscripts $h, v$ indicate the horizontal and vertical directions respectively. Let the sensor path consist of two line segments in parallel with the rectangle sides. The horizontal path is at $y=\bar{y}$ and the vertical path is at $x=\bar{x}$ for some $0 \leq \bar{x} \leq l_{h}, 0 \leq \bar{y} \leq l_{v}$. Let $\Delta x=l_{h} / n_{h}$ and $\Delta y=l_{v} / n_{v}$ be the sampled steps accordingly. The received signal

$$
g\left(\left\|\left(\begin{array}{c}
k \Delta x \\
\bar{y}
\end{array}\right)-y^{*}\right\|\right), g\left(\left\|\left(\begin{array}{c}
\bar{x} \\
k \Delta y
\end{array}\right)-y^{*}\right\|\right)
$$

along the horizontal (vertical) path achieves a (one and only one) local maximum at the unknown $k_{x}$ $\left(k_{y}\right)$ or equivalently at $x_{0}=k_{x} \Delta x\left(y_{0}=k_{y} \Delta y\right)$. Let $m_{h}, m_{v}$ be the given error bounds for the horizontal and vertical directions respectively.

(2) Given a $L$, collect the data

$$
\begin{array}{r}
s_{h j}\left(k-k_{x}\right)=g\left(\left\|\left(\begin{array}{c}
k \Delta x \\
\bar{y}
\end{array}\right)-y^{*}\right\|\right)+v_{h j}(k), \\
j=1,2, \ldots, L, k=0,1, \ldots, n_{h}, \\
s_{v j}\left(k-k_{y}\right)=g\left(\left\|\left(\begin{array}{c}
\bar{x} \\
k \Delta y
\end{array}\right)-y^{*}\right\|\right)+v_{v j}(k), \\
j=1,2, \ldots, L, k=0,1, \ldots, n_{v} .
\end{array}
$$


Define

$$
\begin{aligned}
s_{h}^{L}\left(k-k_{x}\right) & =\frac{1}{L} \sum_{j=1}^{L} s_{h j}\left(k-k_{x}\right) \\
& =g\left(\left\|\left(\begin{array}{c}
k \Delta x \\
\bar{y}
\end{array}\right)-y^{*}\right\|\right)+\bar{v}_{h}(k), \\
s_{v}^{L}\left(k-k_{y}\right) & =\frac{1}{L} \sum_{j=1}^{L} s_{v j}\left(k-k_{y}\right) \\
& =g\left(\left\|\left(\begin{array}{c}
\bar{x} \\
k \Delta y
\end{array}\right)-y^{*}\right\|\right)+\bar{v}_{v}(k) .
\end{aligned}
$$

(3) Determine the estimates $\hat{k}_{x}$ and $\hat{k}_{y}$ of $k_{x}$ and $k_{y}$ respectively by

$\hat{k}_{x}=\operatorname{argmax}_{k} s_{h}^{L}\left(k-k_{x}\right), \hat{k}_{y}=\operatorname{argmax}_{k} s_{v}^{L}\left(k-k_{y}\right)$.

The estimate of $y^{*}$ is given by $\hat{y}=\left(\begin{array}{l}\hat{k}_{x} \Delta x \\ \hat{k}_{y} \Delta y\end{array}\right)$.

(4) Define $n_{h 1}=2 m_{h}+1, n_{v 1}=2 m_{v}+1, n_{h 2}=n_{h}-$ $2 m_{h}-1$ and $n_{v 2}=n_{v}-2 m_{v}-1$. Let $\widehat{s h 1}=s_{h}^{L}\left(\hat{k}_{x}+\right.$ $\left.m_{h}\right), \widehat{s h 2}=\left(s_{h}^{L}\left(\hat{k}_{x}+m_{h}\right)-s_{h}^{L}\left(n_{h}\right)\right) / 2+s_{h}^{L}\left(n_{h}\right)$ and $\widehat{s v 1}=s_{v}^{L}\left(\hat{k}_{y}+m_{v}\right), \widehat{s v 2}=\left(s_{v}^{L}\left(\hat{k}_{y}+m_{v}\right)-\right.$ $\left.s_{v}^{L}\left(n_{v}\right)\right) / 2+s_{v}^{L}\left(n_{v}\right)$. Compute

$$
\begin{array}{r}
p_{h 1}(u)=n_{h 1} \exp \left(-\exp \left(-\frac{\frac{u-\widehat{s h 1}}{\sigma / \sqrt{L}}-a_{n_{h 1}-1}}{b_{n_{h 1}-1}}\right)\right) \\
\cdot \frac{1}{\sqrt{2 \pi \sigma^{2} / L}} e^{-\frac{(u-\widehat{s h 1})^{2}}{2 \sigma^{2} / L}}, \\
p_{h 2}(w)=n_{h 2} \exp \left(-\exp \left(-\frac{\frac{w-\widehat{s h 2}}{\sigma / \sqrt{L}}-a_{n_{h 2}-1}}{b_{n_{h 2}-1}}\right)\right) \\
\cdot \frac{1}{\sqrt{2 \pi \sigma^{2} / L}} e^{-\frac{(w-\widehat{s h 2})^{2}}{2 \sigma^{2} / L}},
\end{array}
$$

and normalize $p_{h 1}$ and $p_{h 2}$,

$$
\begin{aligned}
& p_{h 1}(u)=\frac{1}{\int p_{h 1}(u) d u} p_{h 1}(u), \\
& p_{h 2}(u)=\frac{1}{\int p_{h 2}(u) d u} p_{h 2}(u) .
\end{aligned}
$$

Similarly compute

$$
\begin{array}{r}
p_{v 1}(u)=n_{v 1} \exp \left(-\exp \left(-\frac{\frac{u-\widehat{s v 1}}{\sigma / \sqrt{L}}-a_{n_{v 1}-1}}{b_{n_{v 1}-1}}\right)\right) \\
\cdot \frac{1}{\sqrt{2 \pi \sigma^{2} / L}} e^{-\frac{(u-\widehat{s v 1})^{2}}{2 \sigma^{2} / L}}, \\
p_{v 2}(w)=n_{v 2} \exp \left(-\exp \left(-\frac{\frac{w-\widehat{s v 2}}{\sigma / \sqrt{L}}-a_{n_{v 2}-1}}{b_{n_{v 2}-1}}\right)\right) \\
\cdot \frac{1}{\sqrt{2 \pi \sigma^{2} / L}} e^{-\frac{(w-\widehat{s v 2})^{2}}{2 \sigma^{2} / L}},
\end{array}
$$

and normalize $p_{v 1}$ and $p_{v 2}$,

$$
\begin{aligned}
& p_{v 1}(u)=\frac{1}{\int p_{v 1}(u) d u} p_{v 1}(u), \\
& p_{v 2}(u)=\frac{1}{\int p_{v 2}(u) d u} p_{v 2}(u) .
\end{aligned}
$$

(5) Calculate

$$
\begin{aligned}
& \hat{\rho}_{x}=\iint_{u>w} p_{h 1}(u) p_{h 2}(w) d u d w \\
& \hat{\rho}_{y}=\iint_{u>w} p_{v 1}(u) p_{v 2}(w) d u d w .
\end{aligned}
$$

(6) If the probability $\hat{\rho}_{x} \cdot \hat{\rho}_{y}$ is acceptable, stop. This is an estimated probability of $\left|\hat{k}_{x}-k_{x}\right| \leq m_{h}$ and $\left|\hat{k}_{y}-k_{y}\right| \leq m_{v}$ or equivalently $\left|\hat{k}_{x} \Delta x-x_{0}\right| \leq m_{h} \Delta x$ and $\left|\hat{k}_{y} \Delta y-y_{0}\right| \leq m_{v} \Delta y$. Otherwise increase $L$ and go back to Step 2.

\subsection{An example}

To illustrate the performance of the estimate $\hat{y}$ of $y^{*}$ and its associated probability estimate $\hat{\rho}_{x} \cdot \hat{\rho}_{y}$ with respect to true but unknown $\rho_{x} \cdot \rho_{y}$ for a given error bound $m$, we consider a square area with the side length $l=$ 400 (units). For both directions, $\Delta x=\Delta y=0.1$ or $n_{h}=$ $n_{v}=4,000$. The unknown source is at $y^{*}=\left(\begin{array}{l}200 \\ 195\end{array}\right)$ and the sensor path consists of two lines $\left(\begin{array}{c}k \Delta x \\ 175\end{array}\right)$ and $\left(\begin{array}{l}180 \\ k \Delta y\end{array}\right)$ for $k=0,1, \ldots, 4000$. The signal to noise ratios (SNR) are defined as

$$
S N R_{h}=10 \log \frac{\sum g_{h}(k)^{2}}{\sum v_{h}(k)^{2}}, S N R_{v}=10 \log \frac{\sum g_{v}(k)^{2}}{\sum v_{v}(k)^{2}} .
$$




\begin{tabular}{|c|c|c|c|c|}
\hline \multicolumn{5}{|c|}{$\hat{\rho}_{x} \cdot \hat{\rho}_{y},\left(\right.$ estimation variance) and the true but unknown $\rho_{x} \cdot \rho_{y}\left(l_{h}=l_{v}=400(\right.$ unit $\left.), \Delta x=\Delta y=0.1\right)$} \\
\hline & $\Delta x \cdot m=\Delta y \cdot m=8$ & $\Delta x \cdot m=\Delta y \cdot m=10$ & $\Delta x \cdot m=\Delta y \cdot m=15$ & $\Delta x \cdot m=\Delta y \cdot m=20$ \\
\hline \multicolumn{5}{|c|}{$S N R_{h}=S N R_{v}=-3 d B$} \\
\hline$L=3$ & $.7718(.0752), .8740$ & $.7609(.0765), .9640$ & $.6712(.1015), .9990$ & $.5676(.1109), .9990$ \\
\hline$L=5$ & $.9326(.0239), .9370$ & $.9106(.0314), .9890$ & $.8185(.0642), 1.0000$ & $.0708(.0967), 1.0000$ \\
\hline$L=10$ & $.9947(.0012), .9950$ & $.9935(.0018), 0.9990$ & $.9680(.0125), 1.0000$ & $.9104(.0340), 1.0000$ \\
\hline \multicolumn{5}{|c|}{$S N R_{h}=S N R_{v}=0 d B$} \\
\hline$L=3$ & $.9533(.0143), .9650$ & $.9467(.0176), .9840$ & $.8903(.0368), 1.0000$ & $.7860(.0709), 1.0000$ \\
\hline$L=5$ & $.9959(.0010), .9910$ & $.9945(.0011), 1.0000$ & $.9656(.0133), 1.0000$ & $.9097(.0327), 1.0000$ \\
\hline$L=10$ & $1.0000(0), 1.0000$ & $.9999(0), 1.0000$ & $.8903(.0368), 1.0000$ & $.9927(.0014), 1.0000$ \\
\hline \multicolumn{5}{|c|}{$S N R_{h}=S N R_{v}=5 d B$} \\
\hline$L=3$ & $1.0000(0), 1.0000$ & $1.0000(0), 1.0000$ & $.9984(.0002), 1.0000$ & $.9917(.0012), 1.0000$ \\
\hline$L=5$ & $1.0000(0), 1.0000$ & $1.0000(0), 1.0000$ & $1.0000(0), 1.0000$ & $.9993(.0001), 1.0000$ \\
\hline$L=10$ & $1.0000(0), 1.0000$ & $1.0000(0), 1.0000$ & $1.0000(0), 1.0000$ & $1.0000(0), 1.0000$ \\
\hline
\end{tabular}

Table 2

The estimated probability $\hat{\rho}_{x} \cdot \hat{\rho}_{y}$, its variance in parenthesis based on 1000 Monte Carlo runs and the true $\rho_{x} \cdot \rho_{y}$.

For a given error bound $\left|k_{x}-\hat{k}_{x}\right|,\left|k_{y}-\hat{k}_{y}\right| \leq m=\left(m_{h}=\right.$ $\left.m_{v}\right)$ or equivalently $\left|x_{0}-\hat{k}_{x} \Delta x\right|,\left|y_{0}-\hat{k}_{y} \Delta y\right| \leq 0.1 m$ and a $L$, Table 2 shows $\hat{\rho}_{x} \cdot \hat{\rho}_{y}$, the true but unknown $\rho_{x} \cdot \rho_{y}$ and the estimation variance in parenthesis for different combinations of SNR, $m$ and $L$.

Admittedly, Table 2 is not easy to understand. We give some explanation by referring to Figure 6 . For given $L, m$, the signal to noise ratio $S N R_{h}$ and the measurements collected, there exists a (true but unknown) probability $\rho_{x} \cdot \rho_{y}$ that $y^{*}$ lies in the square centered at the estimate $\hat{y}=\left(\hat{y}_{1}, \hat{y}_{2}\right)^{\prime}$ bounded by $\hat{y}_{1} \pm m \Delta x$ and $\hat{y}_{2} \pm m \Delta y$ in the horizontal and vertical directions respectively. A problem is that the true probability $\rho_{x} \cdot \rho_{y}$ is unknown. The algorithm in this section provides a reliable estimate $\hat{\rho}_{x} \cdot \hat{\rho}_{y}$. For instance, let $L=5, m=8$ and $S N R_{h}=-3 d B$, the actual (but unknown) probability that $y^{*}$ is in the square is 0.9370 as shown in Table 2. The estimated probability is 0.9326 , as in Table 2 , with the estimation variance 0.0239. Simply put, with the given $L, m, S N G_{h}, y^{*}$ is in the square with an estimated probability 0.9326 . If this probability is too low and not acceptable, $L$ can be increased. Let $L=10$. The actual (but unknown) probability is 0.9950 and its estimate is 0.9947 with the estimation variance 0.0012 .

The estimates and variances in parentheses are based on 1000 Monte Carlo simulations. From Table 2, the probability estimates $\hat{\rho}_{x} \cdot \hat{\rho}_{y}$ are reasonable, usually the lower bound as expected since the mean values in the intervals $\left[k_{x}-m, k_{x}+m\right]$ and $\left[k_{y}-m, k_{y}+m\right]$ were taken by the smallest values in the intervals in the derivation of the estimate. When SNRs are not too low at $0 \mathrm{~dB}$ or $5 \mathrm{~dB}$ ranges or $L=5$ or $L=10$, the estimates are actually very close to the true ones. This method provides a practical and reliable way to check if $L, m$ are reason- ably chosen.

\section{$5 \quad$ Spatial averages}

The idea of the last section is based on repeated measurements to average out the effect of noise. The idea of this section is to use spatial averages. As defined before, let the sensor path consist of line segments in parallel to the axes. Because of similarities, we focus on the horizontal path only.

Let the path length be $l$ (units) and the received signal again be

$$
\begin{aligned}
s_{h}\left(k-k_{x}\right) & =s_{h}\left(\left(k-k_{x}\right) \Delta x_{n}\right) \\
& =g\left(\left\|\left(\begin{array}{c}
k \Delta x_{n} \\
\bar{y}
\end{array}\right)-y^{*}\right\|\right)+v(k) \\
& =g_{h}(k)+v(k)
\end{aligned}
$$

where $\Delta x_{n}=l / n$ is supposed to be a design variable. Obviously $n \rightarrow \infty$ implies $\Delta x_{n} \rightarrow 0$. Let $g\left(\left\|\left(\begin{array}{l}x \\ \bar{y}\end{array}\right)-y^{*}\right\|\right)$ achieve a (one and only one) local maximum at $x_{0}=k_{x}(n) \Delta x_{n}$, where $k_{x}(n)$ and $\Delta x_{n}$ are functions of $n$ but $x_{0}$ is fixed. The goal is to find an estimate $\hat{x}_{0}$ of $x_{0}$ or equivalently $\hat{k}_{x}$ of $k_{x}$ for a given $n$.

To allow spatial averages on $s_{h}\left(k-k_{x}\right)$, define

$$
f(k)=f\left(k \Delta x_{n}\right)= \begin{cases}a \cdot k \Delta_{n}+b, & k<0, \\ -a \cdot k \Delta_{n}+b, & k \geq 0,\end{cases}
$$


for some constants $a, b>0$.

The following lemma is an easy exercise.

Lemma 5.1 Consider the function $f(\cdot)$ defined in (21). Then,

$$
\begin{gathered}
f(0)>f(k), f(k)=f(-k), \quad \forall k \text { and } \\
2 f(k) \geq f(k-j)+f(j+k), \quad \forall k, j .
\end{gathered}
$$

Now define the estimate $\hat{k}_{x}$ of $k_{x}$ for a given $n$ as

$$
\hat{k}_{x}=\arg \max _{i} \frac{1}{n} \sum_{k=0}^{n} s_{h}\left(k-k_{x}\right) f(k-i) .
$$

The intuition can be explained as follows. In the absence of noise,

$$
\begin{aligned}
\sum s\left(k-k_{x}\right) f(k-i)= & \sum g_{h}(k) f(k-i) \\
= & g_{h}\left(k_{x}\right) f\left(k_{x}-i\right) \\
& +g_{h}\left(k_{x}+1\right) f\left(k_{x}+1-i\right)+\cdots \\
& +g_{h}\left(k_{x}+j\right) f\left(k_{x}+j-i\right)+\cdots \\
& +g_{h}\left(k_{x}-1\right) f\left(k_{x}-1-i\right)+\cdots \\
& +g_{h}\left(k_{x}-j\right) f\left(k_{x}-j-i\right)+\cdots .
\end{aligned}
$$

Thus, for $i \neq k_{x}$,

$$
\begin{aligned}
& \sum g_{h}(k) f\left(k-k_{x}\right)-\sum g_{h}(k) f(k-i) \\
= & g_{h}\left(k_{x}\right)\left(f(0)-f\left(k_{x}-i\right)\right)+\sum g_{h}\left(k_{x}+k\right) \\
& \cdot\left(f(k)+f(-k)-f\left(k_{x}+k-i\right)-f\left(k_{x}-k-i\right)\right) \\
= & g_{h}\left(k_{x}\right)\left(f(0)-f\left(k_{x}-i\right)\right)+\sum g_{h}\left(k_{x}+k\right) \\
& \cdot\left(2 f(k)-f\left(k+\left(k_{x}-i\right)\right)-f\left(k-\left(k_{x}-i\right)\right)\right)>0
\end{aligned}
$$

by the properties of $f(\cdot)$. This implies that the maximum of $(22)$ is indeed achieved at $i=k_{x}$. To show the convergence of $\hat{k}_{x}$ to $k_{x}$ in the presence of noise, we need to make a technical assumption on $g_{h}(k)=$ $g\left(\left\|\left(\begin{array}{c}k \Delta x_{n} \\ \bar{y}\end{array}\right)-y^{*}\right\|\right)$.

Assumption 5.1 Given $l$ and $\Delta x_{n}=l / n$, let $B(n)>0$ be an integer such that

$$
g_{h}\left(k-k_{x}\right)= \begin{cases}\neq 0, & |k| \leq B(n), \\ 0, & |k|>B(n),\end{cases}
$$

and

$$
0<B(n) \leq k_{x}(n) \leq n-B(n)
$$

where $k_{x}=\arg \max _{k} g_{h}(k)$.
The interpretation is that there exists a bandwidth $B(n)>0$ and outside the bandwidth $\left|k-k_{x}\right|>B(n)$, the received signal $g_{h}\left(k-k_{x}\right)$ is zero. This is a reasonable assumption because in most applications, the received signal strength is inversely proportional to the distance. Hence the received signal strength decays very quickly and is virtually zero if a sensor is not close the source. The assumption also dictates that the source location should be away from the boundary 0 or $l$.

Theorem 5.1 For given $l, n$ and $\Delta x_{n}$, let $x_{0}=$ $k_{x}(n) \Delta x_{n}$ be a point that $g_{h}(\cdot)$ achieves the maximum and $\hat{x}_{0}=\hat{k}_{x}(n) \Delta x_{n}$ be its estimate. Let $\left|x_{0}-\bar{x}_{0}\right|=\bar{j}(n) \Delta x_{n}$ be any non-zero error bound, where $\bar{j}(n)$ and $\Delta x_{n}$ are functions of $n$ but $\bar{j}(n) \Delta x_{n}$ is fixed. Then, the probability that the estimate $\hat{x}_{0}=\hat{k}_{x} \Delta x_{n}$ is within the bound $\bar{j}(n) \Delta x_{n}$ or equivalently $\left|x_{0}-\hat{x}_{0}\right| \leq\left|x_{0}-\bar{x}_{0}\right|$ goes to one as $\Delta x_{n} \rightarrow 0$ or equivalently as $n \rightarrow \infty$.

Proof: Note for any $j$,

$$
\begin{aligned}
& \frac{1}{n} \sum_{k=0}^{n} s_{h}\left(k-k_{x}\right) f(k-j) \\
= & \frac{1}{n} \sum_{k=0}^{n} g_{h}(k) f(k-j)+\frac{1}{n} \sum_{k=0}^{n} v(k) f(k-j) .
\end{aligned}
$$

Let $\left|x_{0}-\hat{x}_{0}\right|=j(n) \Delta x_{n}$. From the first term on the right, we have

$$
\begin{aligned}
& \frac{1}{n} \sum_{k=0}^{n} g_{h}(k) f\left(k-k_{x}\right)-\frac{1}{n} \sum_{k=0}^{n} g_{h}(k) f(k-j) \\
= & \frac{1}{n} g_{h}\left(k_{x}\right)\left(f(0)-f\left(k_{x}-j\right)\right)+\frac{1}{n} \sum_{k=1}^{d} g_{h}\left(k_{x}+k\right) \\
& \cdot\left(2 f(k)-f\left(k+\left(k_{x}-j\right)\right)-f\left(k-\left(k_{x}-j\right)\right)\right) \\
> & \frac{1}{n} g_{h}\left(k_{x}-j\right) \cdot a \cdot\left(\frac{\left|x_{0}-\hat{x}_{0}\right|^{2}}{\Delta x_{n}}-\left|x_{0}-\hat{x}_{0}\right|\right) \\
= & a g_{h}\left(k_{x}-j\right)\left(\left(\frac{\left|x_{0}-\hat{x}_{0}\right|^{2}}{l}-\frac{\left|x_{0}-\hat{x}_{0}\right|}{n}\right)\right. \\
\rightarrow & a g\left(\left\|\left(\begin{array}{c}
x_{0}-\hat{x}_{0} \\
\bar{y}
\end{array}\right)-y^{*}\right\|\right) \frac{\left|x_{0}-\hat{x}_{0}\right|^{2}}{l}>0
\end{aligned}
$$

as $n \rightarrow \infty$. This implies that in the absence of noise, the maximum of (22) is always achieved at $k_{x}$ or equivalently at $\hat{x}_{0}=x_{0}$. The contribution of noise is the second term of (23) in which $f(k-j)$ is bounded for $k \in[0, n]$ and thus

$$
\frac{1}{n} \sum_{k=0}^{n} v(k) f(k-j) \rightarrow 0
$$

in probability as $n \rightarrow \infty$. If follows that for any error bound $\left|x_{0}-\bar{x}_{0}\right|>0$, the probability of $\left|x_{0}-\hat{x}_{0}\right| \leq$ $\left|x_{0}-\bar{x}_{0}\right|$ goes to one. This completes the proof. 
Intuition is that when $n$ increases, there are more and more terms in the optimization (22) that tends to average out the effect of noise better and better.

\section{Concluding remarks}

The only assumption made in this paper is that the received signal strength is monotonic with the distance between the sensor and the source. No structure nor any mathematical description of the signal propagation is assumed or used in the algorithm development. It was shown theoretically that this assumption alone is enough to uniquely determine the source location. Further in the presence of noise, algorithms can be made robust with asymptotic convergence by taking various averages. Though the paper considers only a localization problem in a two dimensional case, the results can be extended to any but finite dimensional case with no or minimal modifications.

The paper only considers the case that there is only one source and its location is fixed during detection. How to extend the results to cases with multiple sources or moving targets will be a future research direction. Another research topic is how to relax Assumption 2.1 so that the results developed in the paper can be extended to a source that the strength is non-radical symmetric.

\section{References}

[1] Bai, E. W., H. Cho, Y. Ye and R. Tempo, (2002), "Optimization with a few violated constraints and its application in bounded error system identification", IEEE Trans on Automatic Control, Vol. 42, pp.1067-1077

[2] Bai, EW, K. Yosief, S. Dasgupta and R. Mudumbai, "Source Location Estimation for Possibly Unknown Propagation Models", Proc of ACC, Portland, OR, USA, June 2014

[3] Bai, EW, H. Baidoo-Williams, R. Mudumbai and S. Dasgupta, "Robust Tracking of Piecewise Linear Trajectories with Binary Sensor Networks", Automatica, 61:134-145, 2015

[4] Baidoo-Williams, H., Dasgupta, S., Mudumbai, R., and Bai, E. W., "On the Gradient Descent Localization of Radioactive Sources", IEEE Signal Processing Letters, pp. 1046 - 1049, 2013.

[5] Bishop, A.N., B. Fidan, B. D. O. Anderson, K. Dogancay and P. N. Pathirana, "Optimality Analysis of Sensor-Target Localization Geometries", Automatica, 46(3):479-492, March 2010 . [6] Bishop, A.N. "Transmitter
power estimation for uncooperative emitters with the CayleyMenger determinant", Proc. of the 19th Mediterranean Conference on Control and Automation (MED), 2011.

[7] Bishop,

A.N., and Pubudu N. Pathirana. "Optimal trajectories for homing navigation with bearing measurements", Proceedings of the 2008 International Federation of Automatic Control Congress, 2008
[8] Bishop, A. N., and Patric Jensfelt. "An optimality analysis of sensor-target geometries for signal strength based localization", Intelligent Sensors, Sensor Networks and Information Processing (ISSNIP), 2009 5th IEEE International Conference on, 2009

[9] Borkar, V. PROBABILITY THEORY, Springer, 1995

[10] Canudas de Wit,C.,Garin,F.,Fabbiano,R.,Rouchon,P. and Rousseau,A. "Source localization using Poisson integrals.", Proceedings of the 3rd IFAC workshop on distributed estimation and control in networked systems. Santa Barbara,USA, 2010

[11] Cao, M., Anderson, B.D.O., and Morse, A.S., "Sensor network localization with imprecise distance measurements", Systems and Control Letters, Vol. 55, pp. 87-93, 2006.

[12] Chen, J., R. Hudson and K. Yao, "A maximum-likelihood parametric approach to source localization", IEEE ICASSP, pp-3013-3016, Salt Lake, UT, 2001.

[13] Fidan, B, S. Dasgupta and B. D. O. Anderson, “ "Guaranteeing Practical Convergence in Algorithms for Sensor and Source Localization", IEEE Trans SP, Vol. 56, No.9, pp.4458-4469, 2008

[14] Fogel,E. and M. Gavish, "nth-order dynamics target observability from angle measurements" IEEE Transactions on Aerospace and Electronic Systems, Vol. 24, No. 3, pp.305308, 1988.

[15] Galambo, J. THE ASYMPTOTIC THEORY OF EXTREME ORDER STATISTICS, Robert E Krieger Publishing Company, Malabar, Florida, 1987

[16] Cochran, J and M. Krstic, "Nonholonomic Source Seeking With Tuning of Angular Velocity", IEEE Trans on Auitomatic Control, Vol.54, No.4, pp.717-731, 2009

[17] Kump, P., E. W. Bai, K. Chan and W. Eichinger, "Detection of shielded radionuclides from weak and poorly resolved spectra using group positive RIVAL", Radiation Measurements, Vol. 48, pp.18-28, 2013.

[18] Liu, A, J. Bunn and K. Chandy, "Sensor networks for the detection and tracking of radiation and other threats in cities", IPSN, April, Chicago, 2011.

[19] Mao, G, BDO Anderson, and B Fidan. "Path loss exponent estimation for wireless sensor network localization." Computer Networksi, Vol 51. No. 10, 2467-2483, 2007

[20] Martin, R.K., A. S. King, J. Pennington, R. W. Thomas, R. Lenahan, and C. Lawyer, "Modeling and Mitigating Noise and Nuisance Parameters in Received Signal Strength Positioning", IEEE Transactions on Signal Processing, vol. 60, no. 10, Oct 2012, pp. 5451-5463.

[21] Martinez, S. and F. Bullo, "Optimal sensor placement and motion coordination for target tracking", Automatica, Vol. 42, pp.661-668, 2006

[22] Nardone,S.C., A.G. Lindgren, and K.F. Gong, "Fundamental properties and performance of conventional bearings-only target motion analysisi", IEEE Trans. on Automatic Control, Vol.29, No. 9, pp.775-787, 1984.

[23] Vilim, R and R. Klann, "RADRAC: A system for detecting, localizing and tracking radioactive sources in real time", Radiation Measurements and Instrumentation, Vol. 168, pp.61-73, 2009.

[24] Wang, J., W.X. Zhend and T. Chen, "Identification of linear dynamic systems operating in a networked environment", Automatica, Vol.12, pp.2763-2772, 2009 